\title{
A two-rigid block model for sliding gravity retaining walls
}

\author{
Riccardo Conti $^{\mathrm{a}, *}$, Giulia M.B. Viggiani ${ }^{\mathrm{b}}$, Simone Cavallo ${ }^{\mathrm{c}}$ \\ a SISSA - International School for Advanced Studies, Via Bonomea 265, 34136 Trieste, Italy \\ ${ }^{\mathrm{b}}$ Dip. Ingegneria Civile, Università di Roma Tor Vergata, Italy \\ c Università di Roma Tor Vergata, Italy
}

\section{A R T I C L E I N F O}

\section{Article history:}

Received 4 March 2013

Received in revised form

1 August 2013

Accepted 24 August 2013

Available online 1 October 2013

Keywords:

Gravity retaining walls

Permanent displacements

Earthquake

Sliding block

Active earth pressure

\begin{abstract}
A B S T R A C T
This paper presents a new two-rigid block model for sliding gravity retaining walls. Some conceptual limitations of a direct application of Newmark's sliding block method to the case of retaining walls are discussed with reference to a simple scheme of two interacting rigid blocks on an inclined plane. In particular, it is shown that both the internal force between the blocks and their absolute acceleration are not constant during sliding, and must be computed by direct consideration of the dynamic equilibrium and the kinematic constraints for the whole system. The same concepts are extended to the analysis of the active soil wedge-wall system, leading to an extremely simple procedure for computing the relative displacements of the wall when subjected to base accelerations exceeding the critical value. A comparison with the results of numerical analyses demonstrates that the proposed method is capable of describing fully the kinematics of the soil wedge-wall system under dynamic loading. On the contrary, direct application of Newmark's method may lead to inaccurate predictions of the final displacements, in excess or in defect depending on a coefficient which emerges from direct consideration of the dynamic equilibrium of the whole system. This coefficient can be viewed as a corrective factor for the horizontal relative acceleration of the wall, related to the mechanical and geometrical properties of the soil-wall system.
\end{abstract}

(c) 2013 Elsevier Ltd. All rights reserved.

\section{Introduction}

In recent literature several cases are reported of damage to gravity and cantilevered retaining walls due to severe seismic shaking $[9,18]$. Well after the seminal works by Okabe [27] and Mononobe and Matsuo [25], several studies have tackled the problem of computing active dynamic pressures with a theoretical [20,24,29,17], experimental [2] and numerical approach [10,8].

More recently, following Newmark [26] and the pioneering work by Richards and Elms [28], the emphasis has gradually shifted towards the computation of wall displacements, in light of performance based design. For instance, Zeng and Steedman [34], Ling [21], Kim et al. [16], Huang [13], Basha and Babu [3], Huang et al. [14] and Trandafir et al. [31] have considered both a translational and rotational failure mechanism for the wall to compute relative displacements using Newmark's approach. The idea is that of defining a critical value of the acceleration, $a_{c}$ $\left(=k_{c} g\right)$, for an active type of soil-wall failure mechanism, and then computing the displacements of the wall due to a base acceleration $a\left(=k_{h} g\right)$, by direct double integration of the relative acceleration $\left(a-a_{c}\right)$. The underlying assumption is that, when the

\footnotetext{
*Corresponding author. Tel.: + 390403787 479; fax: + 390403787528

E-mail addresses: rconti@sissa.it, riccardo.conti@unicusano.it (R. Conti), viggiani@uniroma2.it (G.M.B. Viggiani), simonecavallo@blu.it (S. Cavallo).
}

wall moves relative to its base, the absolute acceleration of the wall remains constant and equal to its critical value [4]. Only a few authors $[33,23,31]$ apply a corrective factor to the relative acceleration, emerging from direct consideration of the equation of motion of the soil-wall system. More in detail, Zarrabi-Kashani [33] proposes an equation in which $k_{c}$ varies during shaking and an iterative procedure must be adopted to integrate the equation of motion in time; Trandafir et al. [31] use the Bishop simplified slice method to determine $k_{c}$, paying less attention to the kinematic conditions at the soil-wall interface when sliding occurs; Michalowski [23] considers a multi-block mechanism derived from slope stability methods yielding an equation of motion that the same author [12] does not recommend for the analysis of retaining walls, due to the formation of multiple shear bands. As a result, wall displacements are still commonly simply obtained by direct double integration of $\left(k_{h}-k_{c}\right)$.

This work examines the dynamic behaviour of gravity retaining walls with dry cohesionless backfill, accumulating relative displacements solely by sliding on their base. A simple scheme of two frictional rigid blocks resting on an inclined plane is examined first to show that Newmark's approach cannot be used directly to compute the relative displacements of the blocks. More in detail, by considering the dynamic equilibrium of the blocks, it is shown that the assumption that the absolute acceleration during sliding is constant and equal to its critical value is not correct, and this affects the internal force between the two blocks. 
Turning the attention to gravity retaining walls, this implies that it is not possible to examine the dynamic equilibrium of the wall on its own, as the dynamic thrust between the active wedge and the wall is an internal force that cannot be computed without knowledge of the absolute acceleration of the soil wedge. The equations of motion of both the soil wedge and the wall are then derived based on dynamic equilibrium and kinematic conditions of the whole system; the predictions of the model are discussed and compared with the results of numerical analyses.

Once again, Newmark's approach cannot be used directly to evaluate the permanent displacements of the soil-wall system unless a corrective factor for the relative accelerations is introduced in the computation. This factor is independent of the seismic excitation and, for realistic geometries of the wall and mechanical properties, takes values between about 0.7 and 1.1.

In the derivation of the model, the vertical component of the base acceleration is neglected $\left(k_{v}=0\right)$, as this has been proven to have a minor effect on the seismic displacements of gravity retaining walls (see e.g. $[33,13,11]$ ). Also, the work does not examine the effects of shear strength reductions due to strain softening of dense sand on shearing.

\section{A simple two-rigid blocks model on an inclined plane}

Fig. 1 shows the problem under examination, consisting of two frictional rigid blocks resting on a plane with an inclination $\alpha$ on the horizontal. The reference axes, $x$ and $y$, are parallel and orthogonal to the inclined plane. $W_{1,2}, \phi_{1,2}$, and $k_{h}$ are the weights of the two blocks, the friction angles at the interface between the blocks and the plane, and the coefficient of horizontal base acceleration. Moreover, $S$ is the compressive internal force between the two blocks, which are assumed to have perfect rigid smooth contact, and $T_{1,2}$ and $N_{1,2}$ are the shear and normal forces at the contact between the blocks and the plane.

The dynamic equilibrium of each block, in the $x$ and $y$ directions, is given by

$\sum F_{x}=\frac{W}{g} \ddot{u}$

$\sum F_{y}=\frac{W_{\ddot{v}}}{g}$

in which $\Sigma F_{x}$ and $\Sigma F_{y}$ are the sums of all the forces acting on the block, while $\ddot{u}$ and $\ddot{v}$ are the absolute accelerations of the block, in the $x$ and $y$ directions, respectively.

$\ddot{u}=\ddot{u}_{\text {base }}+\ddot{u}_{r}=k_{h} g \cos \alpha+\ddot{u}_{r}$

$\ddot{v}=\ddot{v}_{\text {base }}+\ddot{v}_{r}=-k_{h} g \sin \alpha+\ddot{v}_{r}$

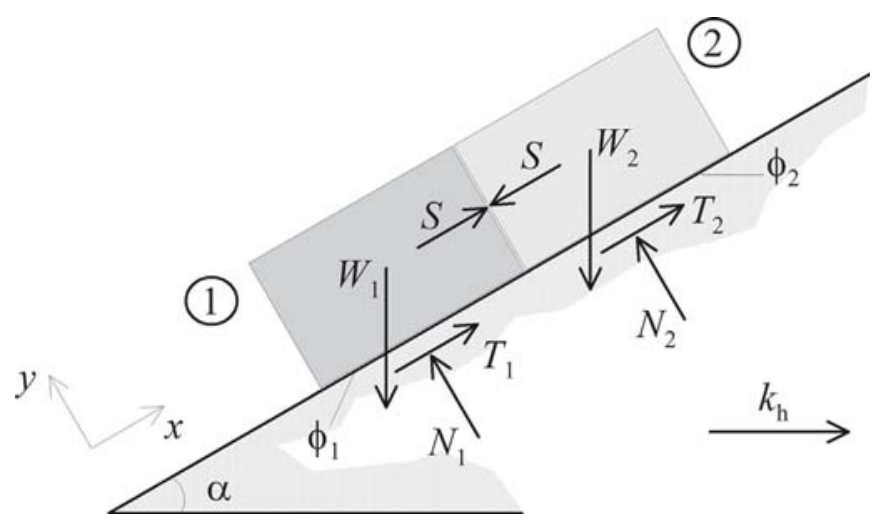

Fig. 1. Two rigid frictional blocks on inclined plane.
Assuming that the two blocks have the same relative displacement in the $x$ direction $\left(u_{1 r}=u_{2 r}=u_{r}\right)$ and thus $\ddot{u}_{1 r}=\ddot{u}_{2 r}=\ddot{u}_{r}$, and that no relative displacements occur in the $y$ direction $\left(v_{1 r}=v_{2 r}=0\right)$ and thus $\ddot{v}_{1 r}=\ddot{v}_{2 r}=0$, Eqs. (1) become

$T_{1}-W_{1}\left(\sin \alpha+k_{h} \cos \alpha\right)-S=W_{1} \frac{u_{r}}{g}$

$N_{1}-W_{1}\left(\cos \alpha-k_{h} \sin \alpha\right)=0$

and

$T_{2}-W_{2}\left(\sin \alpha+k_{h} \cos \alpha\right)+S=W_{2} \frac{u_{r}}{g}$

$N_{2}-W_{2}\left(\cos \alpha-k_{h} \sin \alpha\right)=0$

for block \#1 and block \#2, respectively. Incidentally, note that the condition $v_{r}=0$ implies that the normal forces at the base of the blocks are always positive, which, according to Eqs. (3b) and (4b), requires that

$k_{h}<\frac{1}{\tan \alpha}$

In the following, it is assumed that, even in static conditions, block $\# 2$ is in a limit state, i.e. that $T_{2}=T_{l i m, 2}=N_{2} \tan \phi_{2}$. This assumption, which is necessary to have a unique solution for Eqs. (3) and (4), holds if two conditions are satisfied, namely if $\tan \phi_{2} \leq \tan \alpha$, and $\tan \phi_{1}>\tan \alpha+\left(W_{2} / W_{1}\right)\left(\tan \alpha-\tan \phi_{2}\right)$.

To understand the dynamic behaviour of the system, three conditions have to be examined: (1) the blocks and the base experience the same acceleration $k_{h}<k_{c}$; (2) a limit state (critical) condition is attained in the system, $k_{h}=k_{c}$; and (3) the two blocks start sliding along the base for $k_{h}>k_{c}$.

Until $k_{h}<k_{c}\left(\ddot{u}_{r}=0, T_{1}<T_{\lim 1}\right)$, no relative displacements occur between the blocks and the base, and the internal force between the two blocks is given by

$S=W_{2}\left(\sin \alpha-\cos \alpha \tan \phi_{2}\right)+k_{h} W_{2}\left(\cos \alpha+\sin \alpha \tan \phi_{2}\right)$

which is the sum of a static term proportional to the weight of block \#2 and a dynamic term proportional to the inertia forces acting on the same block.

The critical condition for the system $\left(k_{h}=k_{c}\right.$ and $\left.\ddot{u}_{r}=0\right)$ is attained when all the available friction is mobilized at the base of block \#1, that is when $T_{1}=T_{\lim 1}$. From Eqs. (3) and (4) it is

$k_{c}=\frac{\left(\tan \phi_{1}-\tan \alpha\right)+\left(W_{2} / W_{1}\right)\left(\tan \phi_{2}-\tan \alpha\right)}{\left(1+\tan \phi_{1} \tan \alpha\right)+\left(W_{2} / W_{1}\right)\left(1+\tan \phi_{2} \tan \alpha\right)}$

Thus, the critical acceleration depends on both the mechanical and geometrical properties of the system $\left(\phi_{1,2}\right.$ and $\left.\alpha\right)$ and on the ratio of the weights of the two blocks, while the internal force is given by Eq. (6) with $k_{h}=k_{c}$.

When the applied horizontal acceleration exceeds the critical value $\left(k_{h}>k_{c}\right)$, the two blocks start to slide along the base. The dynamic equilibrium of the system is expressed by

$-\ddot{u}_{r}=\eta\left(k_{h}-k_{c}\right) g$

where

$\eta=\cos \alpha \frac{\left(1+\tan \phi_{1} \tan \alpha\right)+\left(W_{2} / W_{1}\right)\left(1+\tan \phi_{2} \tan \alpha\right)}{1+\left(W_{2} / W_{1}\right)}$

is a coefficient which depends on the mechanical and geometrical properties of the system and on the ratio of the weights of the two blocks. Eq. (8) can be integrated twice, over the time intervals in which the relative velocity, $\dot{u}_{r}$, is non zero, to compute the permanent relative displacements experienced by the two blocks during the dynamic stage. During the same time intervals, the 
internal force is given by

$S=\frac{W_{2}\left(\cos \alpha-k_{h} \sin \alpha\right)}{1+\left(W_{2} / W_{1}\right)}\left(\tan \phi_{1}-\tan \phi_{2}\right)$

Eq. (5) guarantees that the internal force is always positive, but $S$ is not constant, and may be larger or smaller than its critical value $S$ $\left(k_{c}\right)$, depending on the characteristics of the system and on the acceleration time history applied at the base, $k_{h}$. This result clearly arises from the fact that the base is not horizontal; as a matter of fact, if $\alpha=0$, the standard Newmark's approach is recovered, as $S=S\left(k_{c}\right)=$ constant, and $\eta=1$.

The scenario is quite different when the applied base accelerations are leftwards, i.e. when the inertia forces acting on the system would give rise to upwards relative displacements of the two blocks (see Fig. 1). In this case, in fact, the assumption $T_{2}=T_{\lim 2}$ no longer holds if the two blocks are not sliding along the base (upwards or downwards), and the internal force $S$ cannot be computed until upward critical conditions are attained $\left(-k_{h}=k_{c, U P}\right)$. The kinematic quantities for the system, however, can always be defined.

Table 1

Geometrical and mechanical parameters of two-block system.

\begin{tabular}{lccccccc}
\hline & $W_{1}[\mathrm{kN}]$ & $W_{2}[\mathrm{kN}]$ & $\phi_{1}[\mathrm{deg}]$ & $\phi_{2}[\mathrm{deg}]$ & $\alpha[\mathrm{deg}]$ & $k_{c}$ & $\eta$ \\
\hline I & 100 & 50 & 40 & 28 & 30 & 0.11 & 1.23 \\
II & 50 & 100 & 30 & 0 & 0 & 0.19 & 1.00 \\
\hline
\end{tabular}

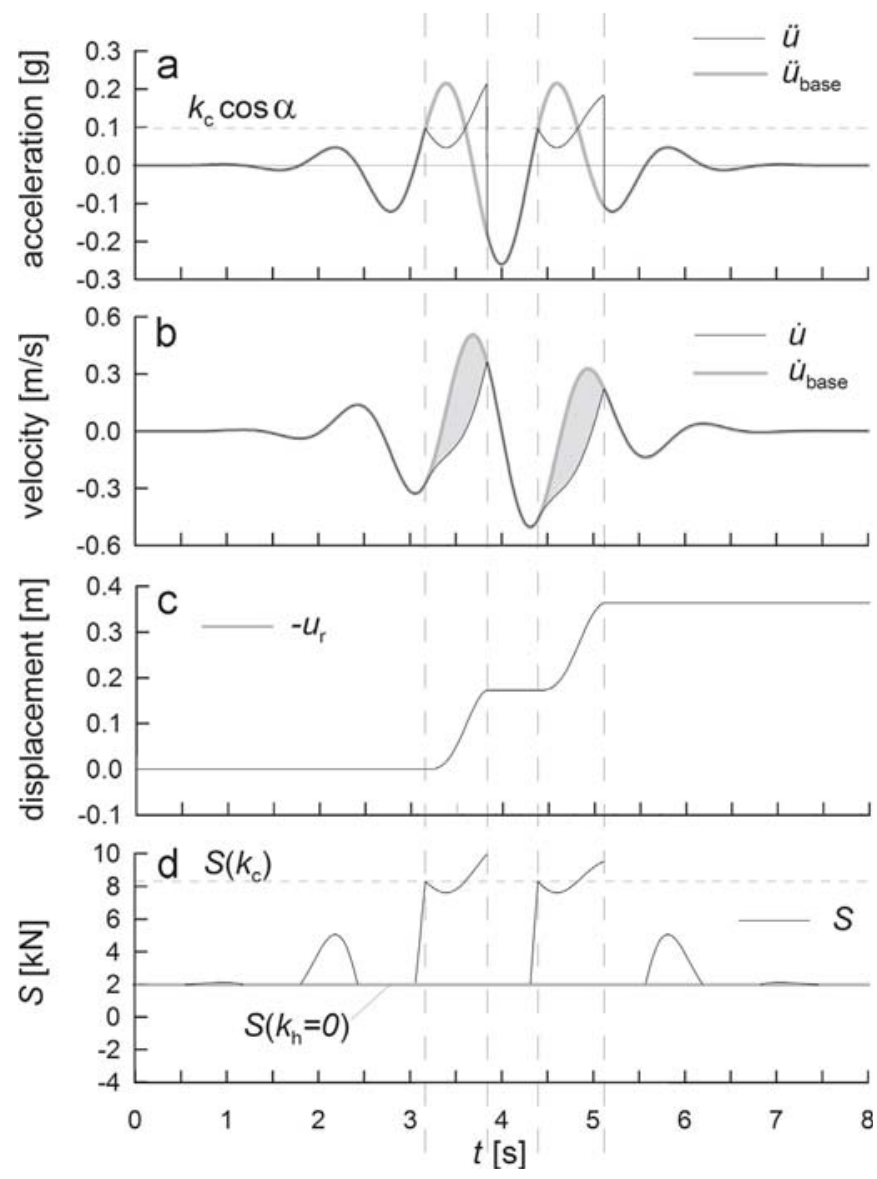

Fig. 2. Two block model - example I: (a) absolute accelerations of the base and the blocks, (b) absolute velocities of the base and the blocks, (c) relative displacements of the blocks, and (d) internal force between the two blocks.

\subsection{Examples}

In order to highlight the main physical implications of the twoblock model outlined above, two examples are discussed here, in which simple excitations are applied at the model base, and the resulting kinematic quantities are computed. Table 1 reports the adopted values of the geometrical and mechanical parameters.

In the first example (I), a base acceleration time history consisting of a wavelet with a maximum acceleration of $0.3 \mathrm{~g}$ is applied at the model base, that has an inclination $\alpha=30^{\circ}$ with respect to the horizontal. The critical seismic coefficients for the system are $k_{c}=0.11$ and $k_{c, U P}=1.60$ for sliding downwards and upwards respectively, thus no upwards relative displacement will take place for the accelerations applied. Fig. 2 shows: (a) the acceleration time history applied at the base, $\ddot{u}_{\text {base }}=k_{h} \cos \alpha$, and the absolute acceleration of the two blocks, $\ddot{u}$; (b) the absolute velocity of the base, $\dot{u}_{\text {base }}$, and of the two blocks, $\dot{u}$; (c) the relative displacement of the two blocks, $u_{r}$, and (d) the internal force between the two blocks, $S$, all in the $x$ direction.

As expected, as far as $k_{h}<k_{c}$, the absolute acceleration of the blocks is equal to that applied at the base. Once the critical condition is attained in the system, the relative velocity between the blocks and the base is non zero and relative displacements occur, which must be computed by double integration of Eq. (8). At the same time, the absolute acceleration and the internal force vary and are both smaller than the corresponding critical values, for $k_{h}>k_{c}$, and larger than the critical values for $k_{h}<k_{c}$. Once the blocks and the base reach the same absolute velocity, a jump occurs in both the absolute acceleration of the blocks and the internal force. However, as emphasized in Fig. 2(d), the rigid-

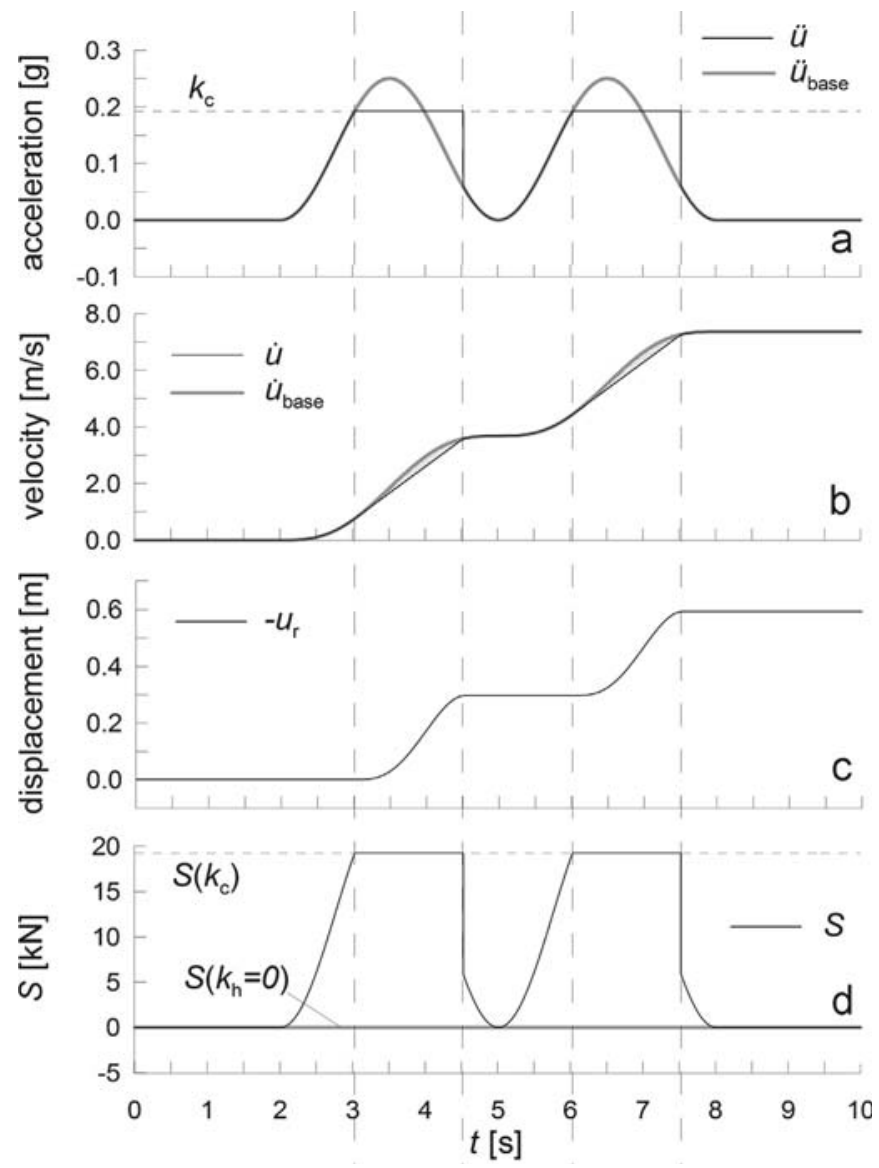

Fig. 3. Two block model - example II: (a) absolute accelerations of the base and the blocks, (b) absolute velocities of the base and the blocks, (c) relative displacements of the blocks, and (d) internal force between the two blocks. 
perfectly plastic model does not allow to compute $S$ in the time intervals in which $k_{h}<0$ and $\dot{u}_{r}=0$.

In the second example (II), the two blocks lie on a horizontal plane and the contact between block \#2 and the base is smooth. A sinusoidal time history of positive acceleration with a maximum of $0.25 \mathrm{~g}$ is applied at the model base, such that inertia forces in the system are always leftwards (see Fig. 3). Once again, relative displacements occur only when the critical condition is attained. However, in this case, both the absolute acceleration of the blocks and the internal force remain constant and equal to the corresponding critical values during all the time intervals in which sliding takes place. This is exactly the conceptual scheme adopted by many authors in the direct application of Newmark's method for the calculation of relative displacements of gravity retaining walls (see e.g., [4]).

\section{Two-rigid block model for sliding gravity retaining walls}

The problem under consideration consists of a dry cohesionless backfill retained by a gravity wall, subjected to a uniform horizontal acceleration $a_{h}=k_{h} g$ directed towards the soil. Fig. 4 shows all the forces and the relevant parameters for the problem, i.e.: the height of the wall, $H$, and the inclination of its internal face, $\beta$; the friction angle of the backfill, $\phi$, the friction angles of the interface between the wall and its base, $\phi_{b}$, and between the wall and the backfill, $\delta$; the slope, $\varepsilon$, and the unit weight, $\gamma$, of the soil backfill. $W_{w}$ and $W$ are the weights of the wall and of the soil wedge, respectively, and $S$ is the internal force between the two, i.e. the soil thrust acting on the wall. Moreover, $T_{w}$ and $N_{w}$ are the shear and normal forces at the base of the wall, and $T_{l i m}$ and $N$ are the shear and normal forces along the slip surface delimiting the soil wedge, which is assumed to be in active limit state in static conditions. This time the $x$ and $y$ directions are taken to be horizontal and vertical i.e., parallel and orthogonal to the base of the wall.

The soil is taken to be rigid-perfectly plastic, and the only considered failure mechanism is sliding of the wall along its base (infinitely strong and rigid foundation).

Following the same procedure as for the two rigid blocks on the inclined plane, and with the kinematic constraints detailed in Appendix A, the equilibrium of forces in the $x$ and $y$ directions is

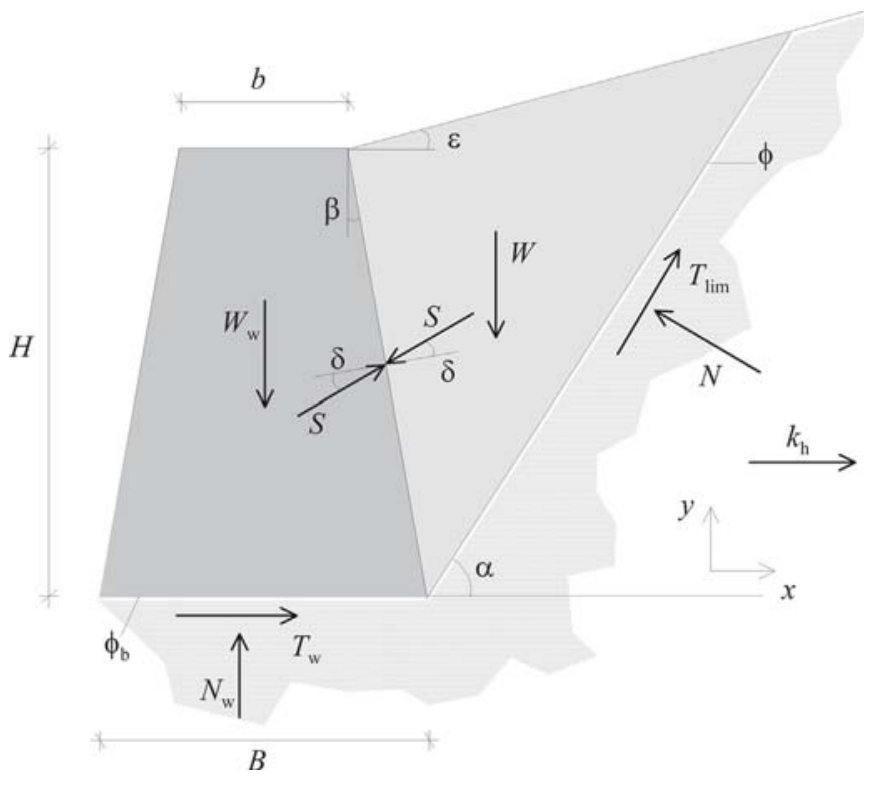

Fig. 4. Retaining wall and active soil wedge. given by (see Fig. 4)

$$
\begin{aligned}
& T_{w}-k_{h} W_{w}-S \cos (\delta+\beta)=W_{w} \frac{\ddot{u}_{r}}{g} \\
& N_{w}-W_{w}-S \sin (\delta+\beta)=0
\end{aligned}
$$

for the wall, and by

$$
\begin{aligned}
& T_{\lim } \cos \alpha-N \sin \alpha-k_{h} W+S \cos (\delta+\beta)=\frac{W}{1+\tan \alpha \tan \beta} \cdot \frac{u_{r}}{g} \\
& T_{\lim } \sin \alpha+N \cos \alpha-W+S \sin (\delta+\beta)=\frac{W \tan \alpha}{1+\tan \alpha \tan \beta} \cdot \frac{u_{r}}{g}
\end{aligned}
$$

for the soil wedge.

As for the two-block model, we will focus our attention on three conditions, namely: (1) the wall-backfill system and the surrounding soil experience the same acceleration $\left(k_{h}<k_{c}\right)$; (2) the limit state (critical condition) is attained in the system $\left(k_{h}=k_{c}\right)$; and (3) the wall (and the soil wedge) starts to slide along its base $\left(k_{h}>k_{c}\right)$.

Condition 1: no sliding $\left(k_{h}<k_{c}\right)$

In the time intervals during which $k_{h}<k_{c} \quad\left(\ddot{u}_{r}=0\right.$ and $\left.T_{w}<T_{\text {lim }, w}\right)$, no relative displacements occur between the wall and the surrounding soil. In this condition, the internal force between the soil wedge and the wall can be computed from Eqs. (12) as

$S(\alpha)=\frac{W}{\cos (\delta+\beta)}\left[\frac{(\tan \alpha-\tan \phi)+k_{h}(1+\tan \phi \tan \alpha)}{(1+\tan \phi \tan \alpha)-\tan (\delta+\beta)(\tan \phi-\tan \alpha)}\right]$

which, again, is the sum of a static term proportional to the weight of the soil wedge and a dynamic term proportional to the inertia forces acting on it. For a given $k_{h}$, the "effective" inclination of the slip surface, $\alpha_{A E}$ [33] and the corresponding soil active thrust, $S_{A E}=S\left(\alpha_{A E}\right)$ [27,25] can be computed by maximizing Eq. (13) with respect to $\alpha$ (see Appendix B).

Condition 2: critical condition $\left(k_{h}=k_{c}\right)$

The critical condition for the system $\left(k_{h}=k_{c}\right.$ and $\left.\ddot{u}_{r}=0\right)$ is attained when all the available friction is mobilized at the base of the wall, that is when $T_{w}=T_{\lim , w}$. By combining Eqs. (11) and (12), the critical seismic coefficient for the wall-soil wedge system can be defined as

$k_{c}^{*}(\alpha)=\frac{\sin \phi_{b} \cos (\delta+\beta+\phi-\alpha)+\left(W / W_{w}\right) \sin (\phi-\alpha) \cos \left(\delta+\beta+\phi_{b}\right)}{\cos \phi_{b} \cos (\delta+\beta+\phi-\alpha)+\left(W / W_{w}\right) \cos (\phi-\alpha) \cos \left(\delta+\beta+\phi_{b}\right)}$

which depends on the mechanical and geometrical properties of the system, on the ratio $W / W_{w}$, and on the inclination $\alpha$ of the slip surface. Again, the effective inclination of the slip surface in critical conditions, $\alpha_{c}$, and the corresponding value of the critical coefficient, $k_{c}=k_{c}^{*}\left(\alpha_{c}\right)$, can be obtained by minimizing Eq. (14) with respect to $\alpha$ (see e.g. [5]). Conceptually, this procedure coincides with finding $k_{c}$ as the pseudostatic coefficient which induces limit equilibrium conditions in the wall, subjected to the inertia forces and the soil active thrust, $S_{A E}$. From Eqs. (11) we get

$k_{c}=\frac{W_{w} \tan \phi_{b}-S_{A E}\left[\cos (\delta+\beta)-\sin (\delta+\beta) \tan \phi_{b}\right]}{W_{w}}$

which corresponds with the implicit equation proposed by Richards and Elms [28] and subsequently adopted in most works in the literature. Thus angle $\alpha_{c}$ can be computed using Eq. (B.2), with $k_{h}=k_{c}$. Unlike Zarrabi-Kashani [33], in this work 
it is assumed that, once the wall starts to slide and as long as relative displacements occur, the slip surface in the backfill does not change. This kinematic assumption, leading to an extremely simple procedure for the solution of the dynamic equation of motion, and more suited to a rigid-block interpretation of the system, will be discussed in the following section comparing the predictions of the model with some numerical results.

\section{Condition 3: sliding $\left(k_{h}>k_{c}\right)$}

In the time intervals during which the horizontal base acceleration is larger than the critical value $\left(k_{h}>k_{c}\right)$, the wall starts to slide along its base. The dynamic equilibrium of the system is still expressed by Eq. (8), in which coefficient $\eta$ can be computed by combining Eqs. (11) and (12)

$\eta=\frac{\cos \phi_{b} \cos (\delta+\beta+\phi-\alpha)+\left(W / W_{w}\right) \cos (\phi-\alpha) \cos \left(\delta+\beta+\phi_{b}\right)}{\cos \phi_{b} \cos (\delta+\beta+\phi-\alpha)+\left(W / W_{w}\right)\left(\cos \phi \cos \beta \cos \left(\delta+\beta+\phi_{b}\right) / \cos (\alpha-\beta)\right)}$

and depends solely on the mechanical and geometrical properties of the system, and on the ratio $W / W_{w}$. In other words, $\eta$ does not depend on the seismic acceleration and can be considered as a correction factor to compute displacements with the classical Newmark sliding block approach. During the same time intervals, the internal force between the soil wedge and the wall is

$S=\frac{W\left(A_{1}+k_{h} A_{2}\right)}{A_{3}+A_{4}\left(W / W_{w}\right)}$

where

$$
\begin{aligned}
& A_{1}=\tan \phi_{b}-\frac{\sin (\phi-\alpha) \cos (\alpha-\beta)}{\cos \phi \cos \beta} \\
& A_{2}=\frac{\sin \alpha \sin (\beta+\phi-\alpha)}{\cos \phi \cos \beta} \\
& A_{3}=\frac{\cos (\alpha-\beta) \cos (\delta+\beta+\phi-\alpha)}{\cos \phi \cos \beta} \\
& A_{4}=\frac{\cos \left(\delta+\beta+\phi_{b}\right)}{\cos \phi_{b}}
\end{aligned}
$$

According to our assumption, both Eqs. (16) and (17) can be computed with reference to $\alpha=\alpha_{c}$.

As already pointed out for the two-block model, the scenario is different when the applied accelerations are leftwards $\left(k_{h}<0\right)$, i.e. when the inertia forces acting on the system are towards the backfill (see Fig. 4). In this case, three different situations can take place, depending on the conditions of the backfill

(i) the wall is still sliding towards the left $\left(\ddot{u}_{r}<0\right)$; in this case, the soil wedge is in active limit state and all the equations presented above apply;

(ii) the relative displacements of the wall are zero $\left(\ddot{u}_{r}=0\right)$; in this case, the backfill is no longer in limit equilibrium conditions and the assumption $T=T_{\lim }$ does not hold. It follows that Eqs. (11)

Table 2

Geometrical properties of the three walls examined in the parametric study.

\begin{tabular}{llll}
\hline Wall & $H[\mathrm{~m}]$ & $B[\mathrm{~m}]$ & $b[\mathrm{~m}]$ \\
\hline A & 4.0 & 2.0 & 1.0 \\
B & 5.0 & 3.0 & 0.5 \\
C & 8.0 & 4.5 & 0.5 \\
\hline
\end{tabular}

and (12) represent an indeterminate system of equations and the internal force $S$ cannot be computed;

(iii) the wall is sliding towards the backfill $\left(\ddot{u}_{r}>0\right)$. In this case, the inertia forces acting on the system induce passive limit state conditions in the backfill $\left(T=T_{\lim }\right)$ and both the internal force $S$ and the relative acceleration $\ddot{u}_{r}$ can be computed by solving the equilibrium equations for the system. This situation would be hardly reached during a real earthquake, when the maximum applied accelerations are usually significantly smaller than the critical value corresponding to which passive conditions are attained in the retained soil.

From the comments above, it is clear that the assumption of rigid-perfectly plastic behaviour for the soil allows for a complete description of all the kinematic quantities of the system, but does not allow for the computation of the dynamic soil thrust during the whole applied acceleration time history. This is of some practical importance if the maximum applied thrust on the wall is of significance, for instance for the structural design of cantilevered retaining walls.

A parametric study was conducted in order to quantify the error associated to a direct application of Newmark's sliding block method to gravity retaining walls, without taking into account coefficient $\eta$ defined in Eq. (16). Table 2 reports the geometry of the three walls examined in the parametric study; three values of $\beta$ $\left(=-10^{\circ}, 0^{\circ}\right.$ and $\left.10^{\circ}\right)$ and two values of $\varepsilon\left(=0^{\circ}\right.$ and $\left.10^{\circ}\right)$ were considered; the friction at the interface between the wall and the soil wedge was always $\delta=2 / 3 \phi$, while the ratio of the friction angle of the retained soil and the friction angle at the wall-base interface, $\phi / \phi_{\mathrm{b}}$, was varied between 0.8 and 1.2. Figs. 5 and 6 show that both the computed values of coefficient $\eta$ and of the critical seismic coefficient, $k_{c}$, increase with the friction angle of the retained soil, $\phi$, and the friction angle of the wall-base interface, $\phi_{b}$. Moreover, $\eta$ increases slightly with increasing $\varepsilon$ and $\beta$, while $k_{c}$ decreases with increasing $\varepsilon$ and decreasing $\beta$. For the range of values considered in the parametric study, $\eta$ varies between about 0.7 and 1.1; it follows that direct application of Newmark's approach may result in a maximum error of $30 \%$.

\section{Numerical investigation}

In order to assess the capability of the proposed model to describe the dynamic behaviour of gravity retaining walls, we discuss the results of two numerical analyses in which different excitations were applied, namely a simple wavelet with a maximum acceleration of $0.3 g$, and a real earthquake registered on rock outcrop (Tolmezzo earthquake: $\mathrm{PGA}=0.35 \mathrm{~g}$, mean period $T_{m}=0.4 \mathrm{~s}$, duration $T_{5-95}=4.2 \mathrm{~s}$ ).

The plane-strain finite difference analyses were carried out on a pair of gravity walls at a distance of $16 \mathrm{~m}$ from one another, retaining a $4 \mathrm{~m}$ thick ideal layer of dry sand (soil \#1) overlying a stiffer soft rock deposit (soil \#2). Fig. 7 shows the finite difference grid adopted in the numerical analyses, with an extension of $100 \mathrm{~m}$, consisting of a total of 8652 elements, with a minimum size of $0.33 \mathrm{~m}$ near the walls. Fig. 8 shows a detail of the grid close to the walls together with the position of three points considered in the discussion of the results: N1, representative of free field conditions; N2, at mid height of the walls; and N3 within the active soil wedges.

The retaining walls were modelled as elastic solid elements with a density $\rho=2.55 \mathrm{mg} / \mathrm{m}^{3}$, a Young's modulus $E=40 \mathrm{GPa}$, and a Poisson's ratio $\nu=0.15$, connected to the grid nodes with elasticperfectly plastic interfaces along both the vertical side $\left(\delta=16^{\circ}\right)$ and the base $\left(\phi_{b}=\phi=25^{\circ}\right)$. 
a

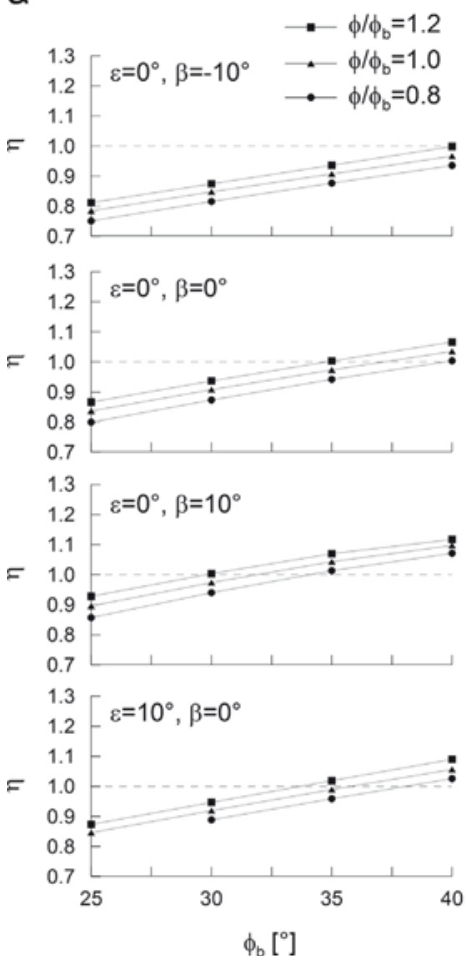

b
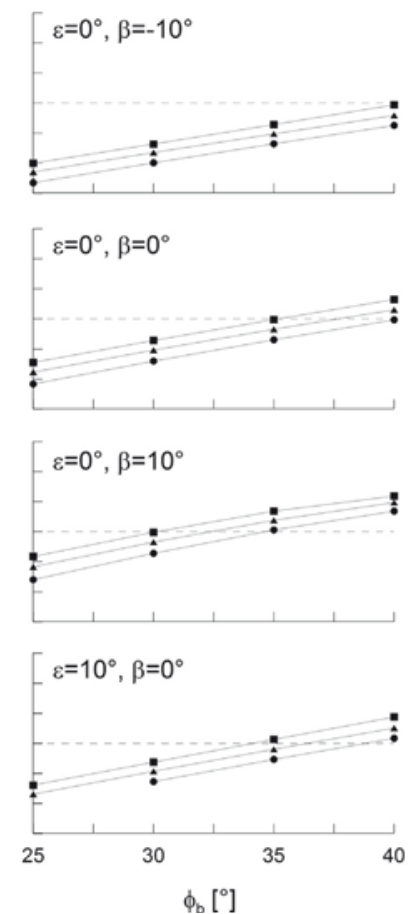
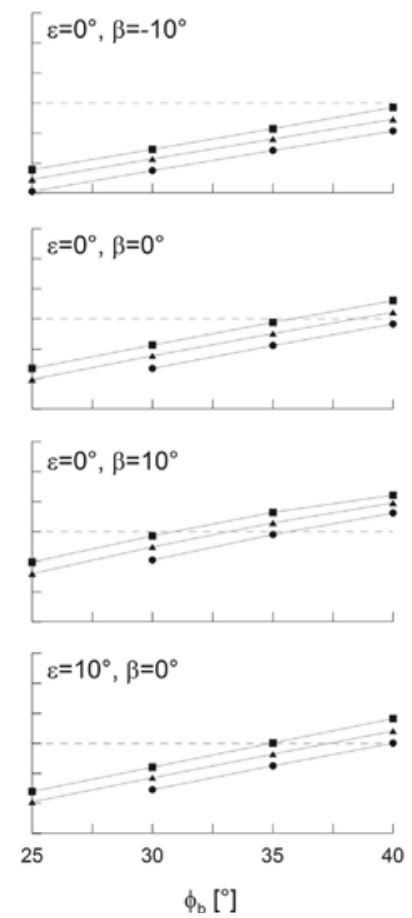

Fig. 5. Computed values of $\eta$ as a function of $\phi_{b}$ for: (a) wall A, (b) wall B, and (c) wall C in Table 2.

a
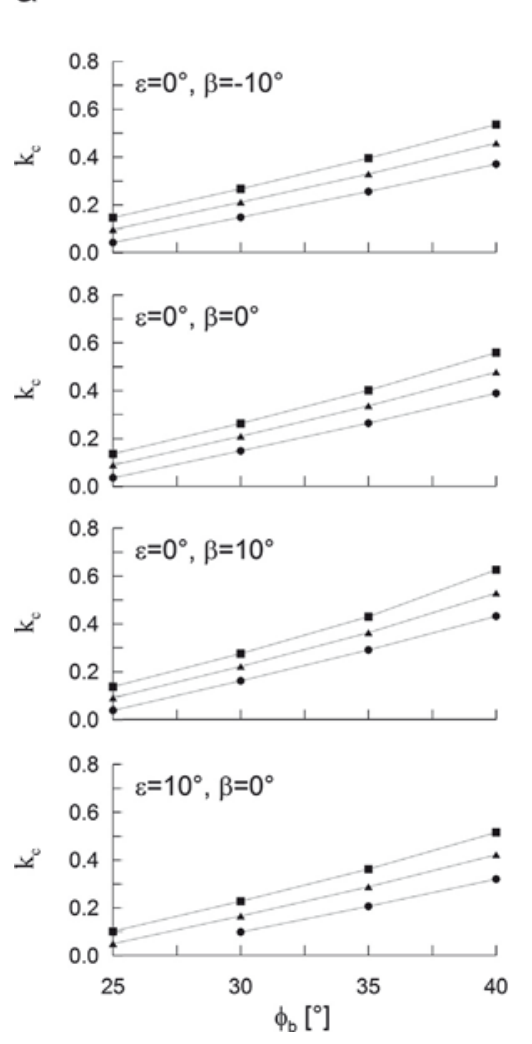

$b$
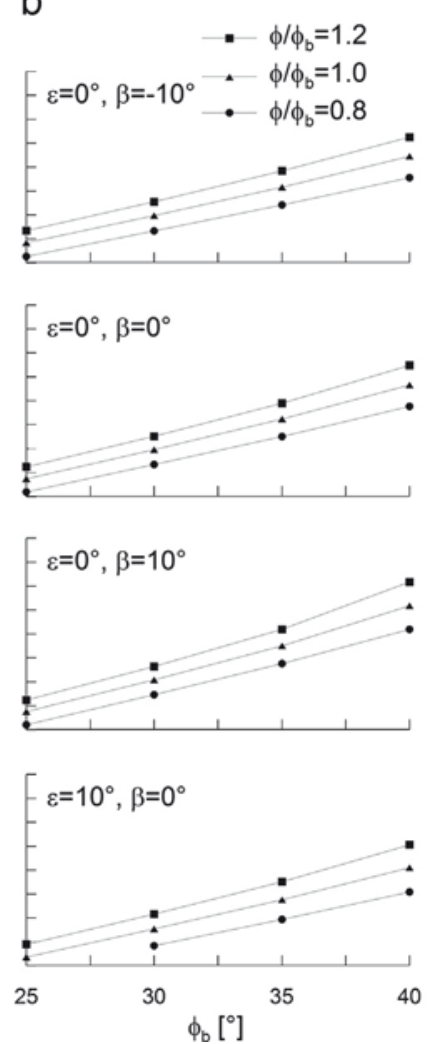

C
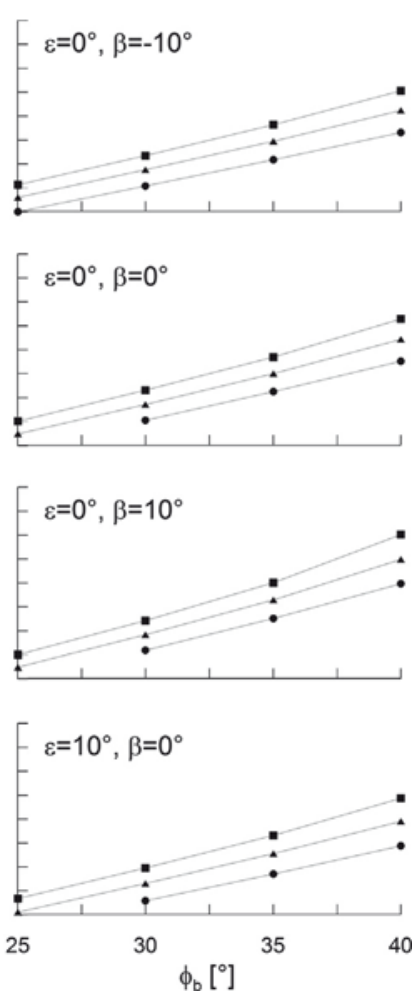

Fig. 6. Computed values of $k_{c}$ as a function of $\phi_{b}$ for: (a) wall A, (b) wall B, and (c) wall C in Table 2 .

The soil was modelled as an elastic-perfectly plastic material with a Mohr-Coulomb failure criterion and a non-associated flow rule with zero dilatancy. The small strain shear modulus is given by $G_{0}=10000 \sqrt{p^{\prime}}(\mathrm{kPa})$ for the retained sand (soil \#1), where $p^{\prime}$ is the mean effective stress, while a constant value of $G_{0}=3.6 \times 10^{3}$ MPa was used for the soft rock layer (soil \#2). In both cases, the Poisson's ratio is $\nu=0.20$. Table 3 summarizes the main physical and mechanical properties adopted in the numerical analyses. 


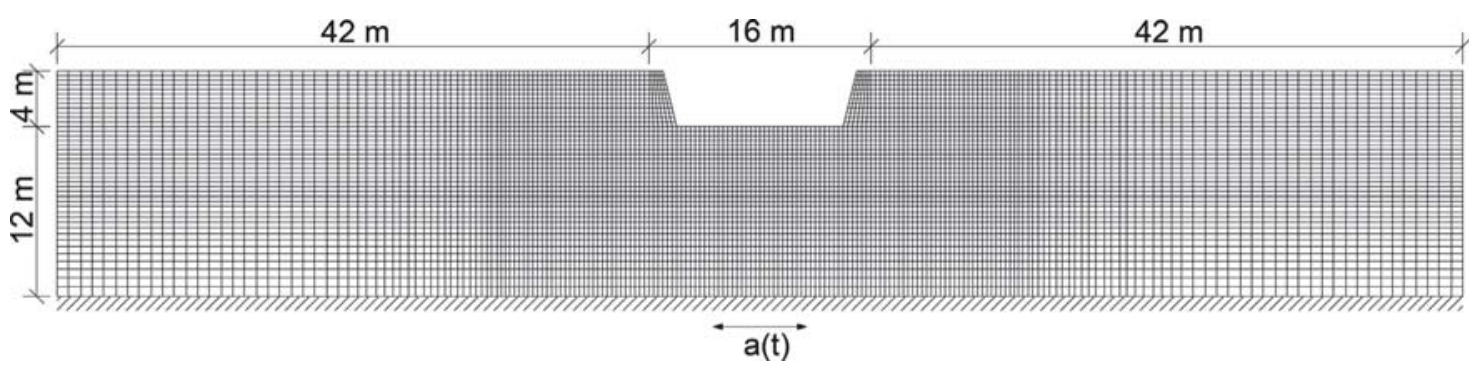

Fig. 7. Finite difference grid adopted in the numerical analyses.

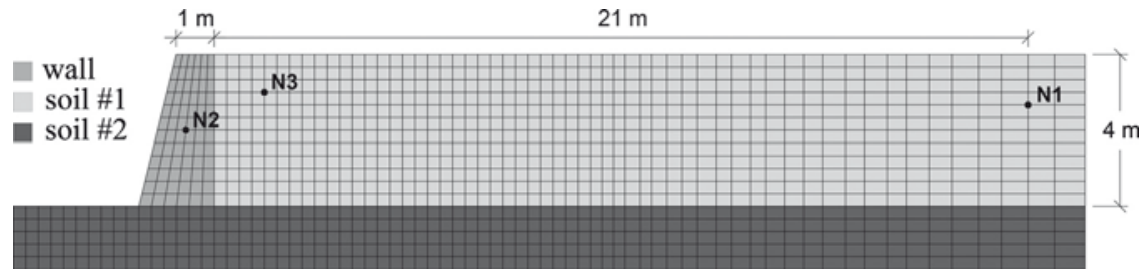

Fig. 8. Detail of the finite difference grid adopted in the numerical analyses showing the points considered in the discussion of results.

Table 3

Physical and mechanical properties adopted in the numerical analyses.

\begin{tabular}{llllll}
\hline Material & $\gamma\left[\mathrm{kN} / \mathrm{m}^{3}\right]$ & $\phi[\mathrm{deg}]$ & $c^{\prime}[\mathrm{kPa}]$ & $G_{0}[\mathrm{MPa}]$ & $K_{0}[\mathrm{MPa}]$ \\
\hline Wall & 25 & - & - & $1.7 \times 10^{4}$ & $1.9 \times 10^{4}$ \\
Soil \#1 & 20 & 25 & 0 & $10.0 \sqrt{ } p^{\prime}$ & $13.3 \sqrt{p^{\prime}}$ \\
Soil \#2 & 20 & 50 & 100 & $3.6 \times 10^{3}$ & $4.9 \times 10^{3}$ \\
\hline
\end{tabular}

For the initial static stage, during which the gravity walls were installed and the backfill elements were activated in successive steps, the shear modulus of the sand layer was set equal to $0.3 \times G_{0}$. During the subsequent dynamic stage, non-linear and dissipative behaviour was introduced for stress paths within the yield surface through a hysteretic model available in the library of FLAC 5.0 [15]. This is an extension to general strain conditions of the one-dimensional non-linear models that make use of the Masing [22] rules to describe the unloading-reloading behaviour of soil during cyclic loading. Fig. 9 shows a comparison between the modulus decay curve and the equivalent damping ratio of the adopted model and that suggested by Vucetic and Dobry [32] for soils with zero plasticity index.

After the static stage, the horizontal acceleration time histories of the input excitations were applied to the bottom nodes of the grid, together with a zero velocity condition in the vertical direction, while standard periodic constraints [35] were applied to the lateral boundaries.

According to the model described above, the critical seismic coefficient of the walls is $k_{c}=0.09$, while coefficient $\eta=0.83$. Fig. 10 shows the results obtained from the numerical analysis with the wavelet input acceleration in terms of computed absolute acceleration of the (a) right and (b) left wall, and (c) relative displacements of right and left wall; these are compared with the predictions obtained with the model described above, using the free field acceleration (N1) as the input base motion. The results show that the absolute acceleration of both walls coincides with the base acceleration until the seismic coefficient is smaller than the critical value, $k_{c}$; once the base acceleration exceeds the critical value, the walls slide on their base under an absolute acceleration that varies with time, both in the numerical and theoretical models, while in Newmark's approach the absolute acceleration of the wall during sliding is taken to be constant and equal to its critical value. The agreement between the numerical and theoretical predictions of the relative displacements of both walls is extremely good, both in magnitude and trend; on the other hand, a prediction obtained by direct application of Newmark's method $(\eta=1)$ over predicts the numerical results by about $17 \%$.

Fig. 11 shows the results from the same analysis, this time in terms of computed absolute (a) horizontal and (b) vertical acceleration of the soil wedge behind the right wall, and absolute (c) horizontal and (d) vertical acceleration of the soil wedge behind the left wall. In the time intervals during which the walls are sliding on their base, the soil wedge is moving with both horizontal and vertical components of acceleration. In the theoretical model, the vertical acceleration of the soil wedge derives from the kinematic constraints detailed in Appendix A, assuming that the inclination of the slip surface remains constant and equal to $\alpha_{c}$; the remarkably good agreement between the numerical predictions and the results confirms that taking $\alpha=\alpha_{c}=$ constant is a reasonable assumption.

Fig. 12 shows the contours of relative horizontal displacements within the retained soil at the two times indicated in Figs. 10 and 11, when the left wall is sliding on its base. For comparison, Fig. 12 also shows the inclination of the critical active soil wedge, $\alpha_{c}=47^{\circ}$. While it is clear that there is no well defined slip surface, but rather a gradual transition to zero relative displacements away from the wall, it is also evident that there is a soil wedge immediately behind the wall which is acting as a rigid body, delimited by a surface at an inclination corresponding closely to $\alpha_{c}$. This result is qualitatively similar to that obtained experimentally by Aitken [1] from small scale shaking table models of retaining walls. Photographs taken at the end of the tests revealed that in the retained soil there were a number of slip surfaces, across a transition zone between a nearly rigid soil wedge and the undisturbed soil.

Finally, Fig. 13 shows the results obtained from the numerical analysis carried out using the Tolmezzo record as input base acceleration, in terms of computed absolute acceleration of the (a) right and (b) left wall, and (c) relative displacements of right and left wall; as before, these are compared with the predictions obtained using both the theoretical model and by direct 


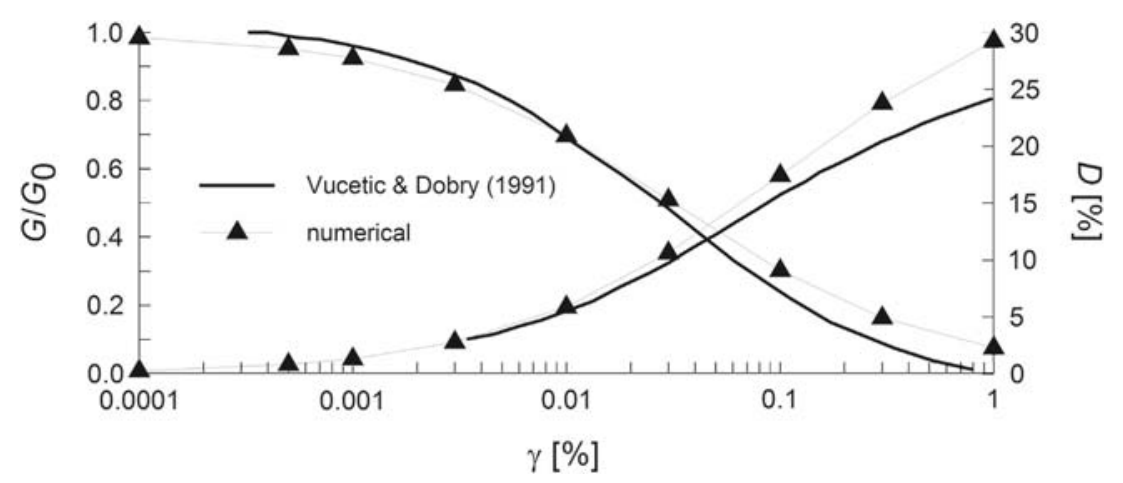

Fig. 9. Modulus decay and damping ratio curves for the constitutive soil model.
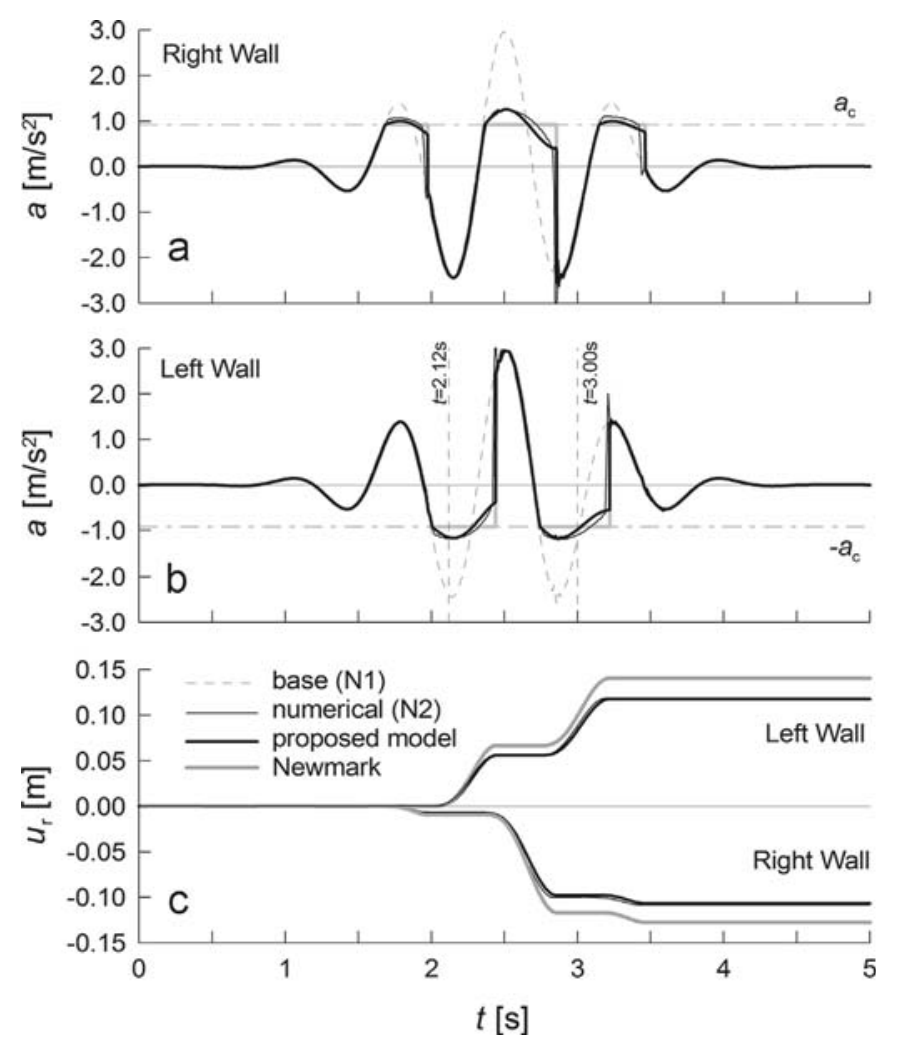

Fig. 10. Wavelet input acceleration. Comparison of numerical and model predictions for: absolute horizontal acceleration of (a) right and (b) left wall; (c) relative displacements of right and left wall.

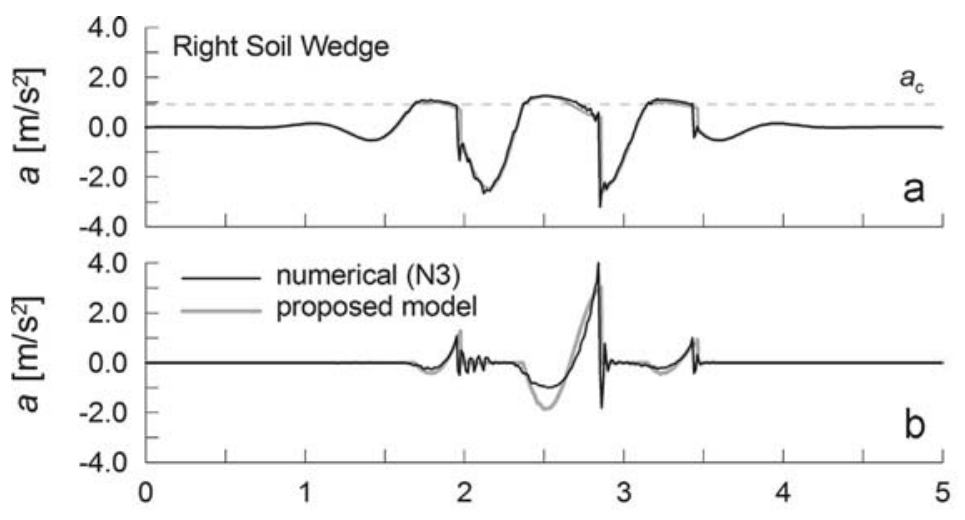

$t[\mathrm{~s}]$

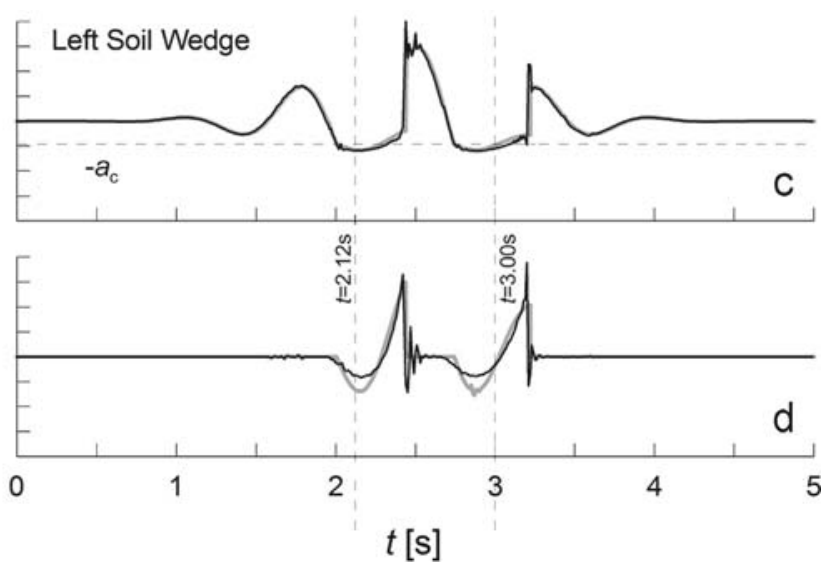

$t$ [s]

Fig. 11. Wavelet input acceleration. Comparison of numerical and model predictions for: absolute (a) horizontal and (b) vertical acceleration of the soil wedge behind the right wall; absolute (c) horizontal and (d) vertical acceleration of the soil wedge behind the left wall. 

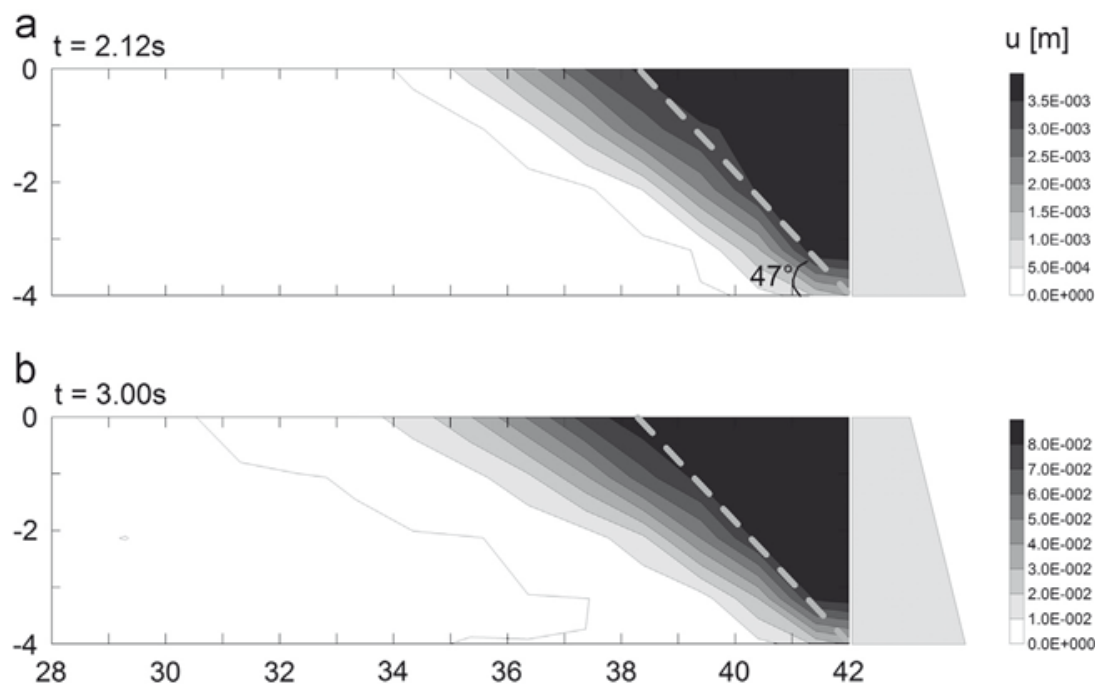

Fig. 12. Wavelet input acceleration. Contours of relative horizontal displacements at two times when the left wall is sliding on its base: (a) $t=2.12 \mathrm{~s}$ and (b) $t=3.00 \mathrm{~s}$.
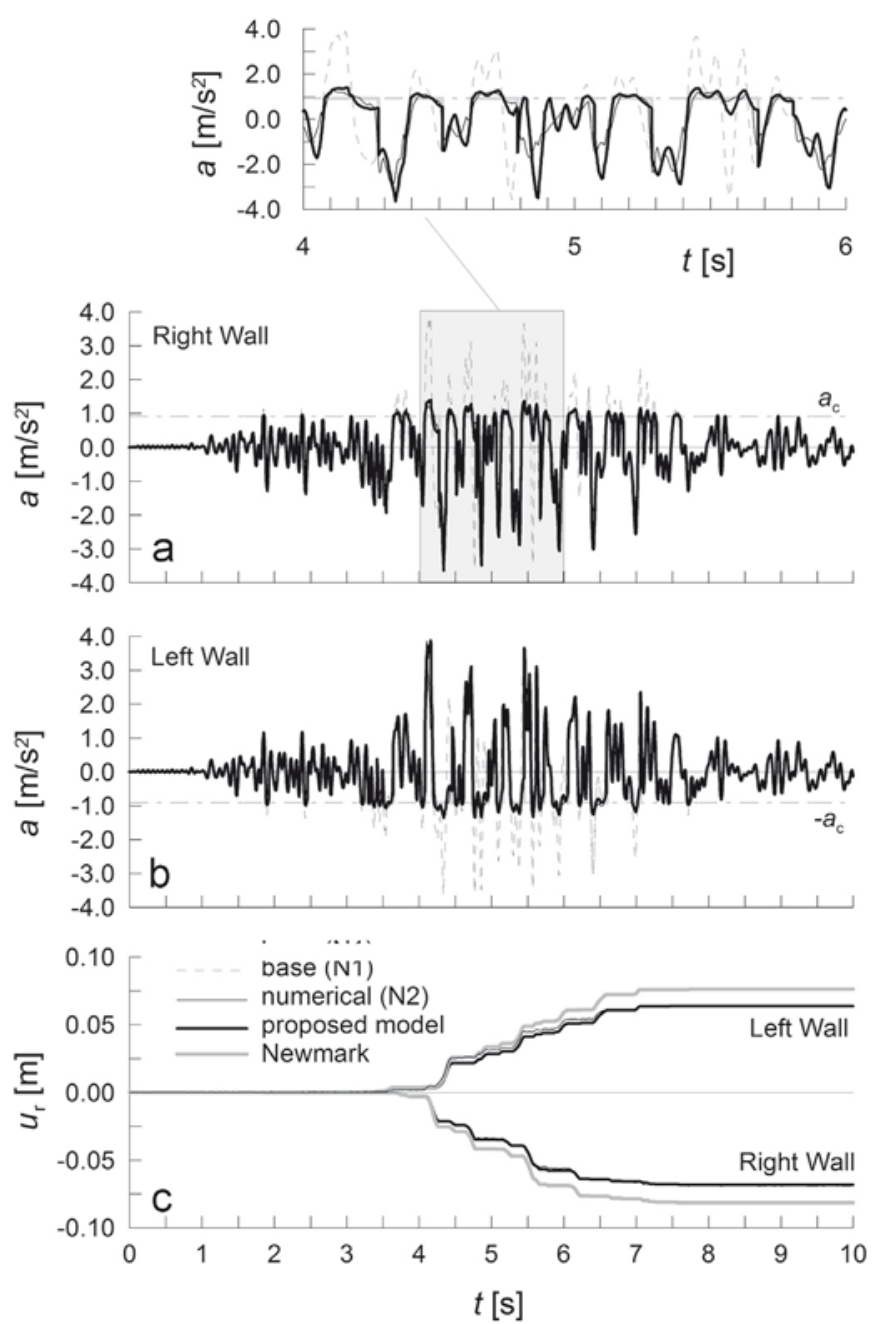

Fig. 13. Tolmezzo input acceleration. Comparison of numerical and model predictions for: absolute horizontal acceleration of (a) right and (b) left wall; (c) relative displacements of right and left wall.

application of Newmark's method. The agreement between the numerical and theoretical predictions of the relative displacements of both walls is extremely good, both in magnitude and trend; once again, the prediction obtained by direct application of Newmark's method over predicts the numerical results by about $17 \%$. 

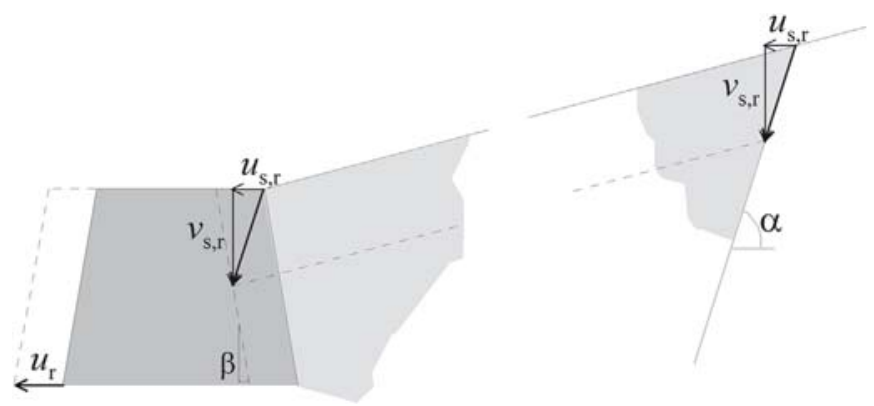

Fig. A1. Kinematic constraints for the soil wedge-wall system during sliding.

\section{Conclusions}

A new two rigid block model for sliding gravity retaining walls has been developed. Some conceptual limitations of direct application of Newmark's sliding block method to the case of retaining walls have been discussed with reference to a simple scheme of two rigid frictional blocks resting on an inclined plane and interacting with one another. In particular, it has been shown that both the internal force between the blocks and their absolute acceleration are not constant during sliding, and must be computed by direct consideration of the dynamic equilibrium and kinematic constraints for the whole system.

The same concepts have been extended to the analysis of the dynamic equilibrium of a soil wedge, in active limit state, interacting with a gravity retaining wall subjected to a base excitation exceeding the critical acceleration of the soil-wall system. The simple assumption that the inclination of the slip surface delimiting the active wedge during sliding does not vary with time yielded an extremely simple procedure to compute the relative displacements of the wall. The comparison with the results of numerical analyses is very encouraging, demonstrating that the kinematic assumption on the inclination of the slip surface is reasonable; moreover, the proposed method is capable of describing fully the kinematics of the whole system under dynamic loading. On the contrary, direct application of Newmark's analysis may lead to inaccurate predictions of the final displacements, in excess or in defect depending on the value taken by $\eta$, which is independent of the seismic acceleration and can be viewed as a corrective factor for the horizontal relative

\section{Appendix A}

If $\ddot{u}_{r}$ and $\ddot{v}_{r}$ are the relative accelerations of the wall in the horizontal and vertical direction, respectively, and $\ddot{u}_{s, r}$ and $\ddot{v}_{s, r}$ are the corresponding relative accelerations of the soil wedge, three kinematic constraints must be satisfied when the wall-soil wedge system starts to experience permanent displacements, i.e.: (1) the wall is sliding on its base, $\ddot{v}_{r}=0 ;(2)$ the soil wedge is sliding on the slip surface, $\ddot{v}_{s, r}=\ddot{u}_{s, r} \tan \alpha$; and (3) the wall and the soil wedge remain in contact while sliding, $\ddot{u}_{s, r}+v_{s, r} \tan \beta=\ddot{u}$ (see Fig. A1). By combining the last two compatibility conditions, one gets

$$
\begin{aligned}
& \ddot{u}_{s, r}=\frac{\ddot{u}_{r}}{1+\tan \alpha \tan \beta} \\
& \ddot{v}_{s, r}=\frac{\ddot{u}_{r} \tan \alpha}{1+\tan \alpha \tan \beta}
\end{aligned}
$$

\section{Appendix B}

The weight of the soil wedge is defined as

$W(\alpha)=\frac{1}{2} \gamma H^{2} \frac{\cos (\beta-\varepsilon) \cos (\alpha-\beta)}{\cos ^{2} \beta \sin (\alpha-\varepsilon)}$

Using Eq. (B.1) and maximizing Eq. (13) with respect to $\alpha$ (or $\tan \alpha$ ), the inclination of the slip surface, for a given $k_{h}$, can be expressed as [33]

$\alpha_{A E}=\phi-\psi+\tan ^{-1}\left[\frac{\tan (\phi-\psi-\varepsilon) \pm C_{1}}{C_{2}}\right]$

where $\psi=\tan ^{-1}\left(k_{h}\right)$, with $\psi \leq \phi-\varepsilon$, and

$C_{1}=\sqrt{\tan (\phi-\psi-\varepsilon) \cdot[\tan (\phi-\psi-\varepsilon)+\cot (\phi-\psi-\beta)] \cdot[1+\tan (\delta+\psi+\beta) \cot (\phi-\psi-\beta)]}$

$C_{2}=1+\tan (\phi+\psi+\beta) \cdot[\tan (\phi-\psi-\varepsilon)+\cot (\phi-\psi-\beta)]$

Eq. (B.2) differs slightly from that proposed by Zarrabi-Kashani [33], and adopted by many authors (see e.g. [19,17]), as both "+ " and "-" are taken into account. As a matter of fact, the appropriate sign must be adopted in order to have $0<\alpha_{A E}<\beta+\pi / 2$.

The soil active thrust acting on the wall can be computed from Eq. (13) and Eq. (B.2) as $S_{A E}=S\left(\alpha_{A E}\right)$, which gives the well known Mononobe-Okabe expression [27,25]:

$S_{A E}=\frac{1}{2} \gamma H^{2} K_{A E}$

where $K_{A E}$ is the dynamic active earth pressure coefficient

$K_{A E}=\frac{\cos ^{2}(\phi-\beta-\psi)}{\cos \psi \cos ^{2} \beta \cos (\delta+\beta+\psi)[1+\sqrt{(\sin (\delta+\phi) \sin (\phi-\varepsilon-\psi) / \cos (\delta+\beta+\psi) \cos (\varepsilon-\beta))}]^{2}}$

acceleration of the wall, related only to the mechanical and geometrical properties of the soil-wall system. A limited parametric study has been conducted to evaluate $\eta$ for realistic geometries of the wall and mechanical properties of the retained soil and the wall-soil and wall-base interfaces, yielding values of $\eta$ between about 0.7 and 1.1 .

\section{Acknowledgements}

The work presented in this paper was developed with the financial support of the Italian Department of Civil Protection within the ReLUIS research project. The Authors wish to acknowledge Prof. Antonio DeSimone and Prof. Massimo Conti for many fruitful discussions during the initial stages of the work.

\section{References}

[1] Aitken GH. Seismic response of retaining walls. MS thesis. Christchurch: University of Canterbury, New Zealand; 1982.

[2] Atik LA, Sitar N. Seismic earth pressures on cantilever retaining structures. Journal of Geotechnical and Geoenvironmental Engineering 2010;136(10):1324-33.

[3] Basha BM, Babu GLS. Computation of sliding displacements of bridge abutments by pseudo-dynamic method. Soil Dynamics and Earthquake Engineering 2009;29(1):103-20.

[4] Callisto L, Soccodato FM. Seismic design of flexible cantilevered retaining walls. Journal of Geotechnical and Geoenvironmental Engineering 2010;136 (2):344-54.

[5] Caltabiano S, Cascone E, Maugeri M. Static and seismic limit equilibrium analysis of sliding retaining walls under different surcharge conditions. Soil Dynamics and Earthquake Engineering 2012;37:38-55.

[8] Evangelista A, Scotto di Santolo A, Simonelli AL. Evaluation of pseudostatic active earth pressure coefficient of cantilever retaining walls. Soil Dynamics and Earthquake Engineering 2010;30(11):1119-28.

[9] Fang YS, Yang YC, Chen TJ. Retaining walls damaged in the Chi-Chi earthquake. Canadian Geotechnical Journal 2003;40:1142-53. 
[10] Gazetas G, Psarropoulos PN, Anastasopoulos I, Gerolymos N. Seismic behaviour of flexible retaining systems subjected to short-duration moderately strong excitation. Soil Dynamics and Earthquake Engineering 2004;24(7):537-50.

[11] Gazetas G, Garini E, Anastasopoulos I, Georgarakos T. Effects of near-fault ground shaking on sliding systems. Journal of Geotechnical and Geoenvironmental Engineering 2009;135(12):1906-21.

[12] Green RA, Michalowski RL. Shear band formation behind retaining structures subjected to seismic excitation. Foundations of Civil and Environmental Engineering 2006;7:157-69.

[13] Huang CC. Seismic displacement of soil retaining walls situated on slope. Journal of Geotechnical and Geoenvironmental Engineering 2005;131 (9):1108-17.

[14] Huang CC, Wu SH, Wu HJ. Seismic displacement criterion for soil retaining walls based on soil strength mobilization. Journal of Geotechnical and Geoenvironmental Engineering 2009;135(1):74-83.

[15] Itasca. FLAC - Fast Lagrangian Analysis of Continua v. 5.0. User's Manual; 2005

[16] Kim SR, Jang IS, Chung CK, Kim MM. Evaluation of seismic displacements of quay walls. Soil Dynamics and Earthquake Engineering 2005;25(6):451-9.

[17] Kim WC, Park D, Kim B. Development of a generalised formula for dynamic active earth pressure. Géotechnique 2010;60(9):723-7.

[18] Koseki J, Koda M, Matsuo S, Takasaki H, Fujiwara T. Damage to railway earth structures and foundations caused by the 2011 off the Pacific Coast of Tohoku Earthquake. Soils and Foundations 2012;52(5):872-89.

[19] Kramer SL. Geotechnical earthquake engineering. Upper Saddle River, New Jersey: Prentice Hall; 1996.

[20] Lancellotta R. Lower-bound approach for seismic passive earth resistance. Géotechnique 2007;57(3):319-21.

[21] Ling HI. Recent applications of sliding block theory to geotechnical design. Soil Dynamics and Earthquake Engineering 2001;21(3):189-97.

[22] Masing G. Eigenspannungen und Verfertigung bim Messing. In: Proceedings of the 2nd international congress on applied mechanics, Zurich; 1926.
[23] Michalowski RL. Displacements of multiblock geotechnical structures subjected to seismic excitation. Journal of Geotechnical and Geoenvironmental Engineering 2007;133(11):1432-9.

[24] Mylonakis G, Kloukinas P, Papantonopoulos C. An alternative to the Mononobe-Okabe equations for seismic earth pressures. Soil Dynamics and Earthquake Engineering 2007;27(10):957-69.

[25] Mononobe N, Matsuo $\mathrm{H}$. On the determination of earth pressure during earthquake. In: Proceedings of the 2nd world engineering conference, vol. 9; 1929. p. $177-85$

[26] Newmark NM. Effects of earthquakes on dams and embankments. Géotechnique 1965;15(2):139-60.

[27] Okabe S. General theory of earth pressure and seismic stability of retaining wall and dam. Journal of Japanese Society of Civil Engineering 1924;12:1.

[28] Richards R, Elms DG. Seismic behaviour of gravity retaining walls. Journal of the Geotechnical Engineering Division, ASCE 1979;105(4)449-64.

[29] Shukla SK, Gupta SK, Sivakugan N. Active earth pressure on retaining wall for $c-\phi$ soil backfill under seismic loading condition. Journal of Geotechnical and Geoenvironmental Engineering 2009;135(5):690-6.

[31] Trandafir AC, Kamai T, Sidle RC. Earthquake-induced displacements of gravity retaining walls and anchored-reinforced slopes. Soil Dynamics and Earthquake Engineering 2009;29(3):428-37.

[32] Vucetic M, Dobry R. Effect of soil plasticity on cyclic response. Journal of Geotechnical and Geoenvironmental Engineering 1991;117(1):89-107.

[33] Zarrabi-Kashani K. Sliding of gravity retaining wall during earthquakes: considering vertical acceleration and changing inclination of failure surface. MS thesis. Department of Civil Engineering, Cambridge: Massachusetts Institute of Technology, Massachusetts; 1979.

[34] Zeng X, Steedman RS. Rotating block method for seismic displacement of gravity walls. Journal of Geotechnical and Geoenvironmental Engineering 2000;126(8):709-17.

[35] Zienkiewicz OC, Bianic N, Shen FQ. Earthquake input definition and the transmitting boundary condition. In: St. Doltnis I, editor. Conference on advances in computational non-linear mechanics; 1988. p. 109-38. 